\title{
Suicide prevention according to different health professionals: quantification analysis in a qualitative study
}

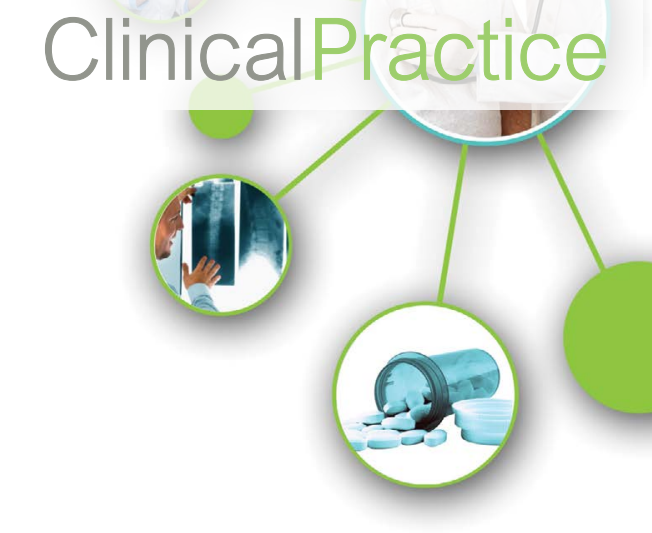

Objective: The purpose of this study is to analyze the differences in the perception of suicide prevention of four different groups of health professionals in a region in north-western Spain.

Methods: Eight focal groups were made (two for each professional category) involving a total of 12 primary care physicians, 14 emergency care physicians, 17 psychologists and 13 psychiatrists. A qualitative analysis was conducted alongside a quantitative analysis to quantify the qualitative data.

Results: Qualitative analysis: The four groups of health professionals attach more importance to intervention in risk behaviors. All of them agree that there are difficulties in intervention, the main one for primary care and emergency care professionals being the lack of training and time, while psychiatrists and psychologists believe the main issue lies in the management of suicidal behavior. Unlike the other groups, psychiatrists attach greater importance to resources and their availability and accessibility. Quantitative analysis: There are clear associations between psychologists and intervention in risk behaviors, primary care physicians and difficulties in suicide prevention, emergency care physicians and the resources available in the area of prevention, and psychiatrists and the work-related relevance they attach to these suicidal behaviors.

Conclusion: All these professionals agree on the need for arranging improved assistance and prevention for people at risk for suicide. Future research is required to make progress in suicide prevention and in the treatment of suicide-related behaviors from the healthcare sector.

\section{KEYWORDS: suicide, behavior, prevention, health professionals}

\section{Introduction}

Suicide is the tenth leading cause of death in the world [1], accounting for around 800,000 deaths in the world each year, the number of people who attempt it being even higher. Thus, the suicide rate is estimated at 11.4 per 100,000 inhabitants (15.0 for men and 8.0 for women) [2].

Consequently, suicide prevention is one of the most relevant issues to public health policies [3], and there are numerous examples of interventions aimed at preventing it. Initiatives for suicide prevention have proved to be generally effective, so that they have the potential to be integrated in the work programs of mental health services [4]. There is also evidence that the inclusion of a suicide prevention program in the primary care network could reduce the number of suicides, especially in rural areas [5]. This has raised the issue of the need for faculties to incorporate specific training in suicide prevention as a major part in medical studies and for the development of medical training programs aimed at early detection and management of suicidal behavior [6]. Finally, attention should be drawn to the increasingly frequent implementation of suicide prevention programs based on the use of new technologies, which have proved effective [7-11].

In terms of prevention, it should be understood that the suicidal process can be defined as a progression of different stages during which the risk of suicide gradually increases. This process begins with thoughts of death or suicide, going through structured suicidal ideation, planning of the act of suicide and, finally, attempting it [12]. This progression from thought to action signifies the transition from mild to severe in the symptomatology of the suicidal process [13]. It should be emphasized that a suicide attempt is the most powerful predictor of future accomplished suicides and it is estimated that about $21 \%$ to $40 \%$ of the
Muñoz-Sánchez $\mathrm{JL}^{* 1}$, Sánchez-Gómez $\mathrm{MC}^{2}$, Martín-Cilleros $\mathrm{MV}^{3}$, Blanco-Dorado $\mathrm{C}^{4}$, Franco-Martín $\mathrm{MA}^{5}$

${ }^{1}$ Department of Psychiatry, Zamora Hospital. Zamora

${ }^{2}$ Directora del departamento Didáctica de investigación y Métodos de investigación. Facultad de Educación. Universidad de Salamanca

${ }^{3}$ Subdirectora de la Escuela de Magisterio de Zamora

${ }^{4}$ Psychiatris, Department of Psychiatry, Zamora Hospital, Zamora

${ }^{5}$ Head of Department of Psychiatry at Zamora Hospital, Associate Professor at the University of Salamanca, Head of RDI Area at INTRAS Foundation

*Author for correspondence:

jlmusa@icloud.com 
individuals that die for this reason have a history of one or more earlier attempts [14]. Although most attempts do not end in death, each of them involves the possibility of serious short- or long-term physical damage, which adds to the psychological suffering caused by living such experiences and increases future risk of this type of behavior [15].

Likewise, the existence or prior mental illness, stressors and certain sociodemographic parameters feature prominently among the numerous identified factors that increase the risk of suicide [16,17]. There is a direct link between stressful events and environmental changes and the risk of suicide [18]. A history of mental illness or its suffering seems to be the greatest risk factor for suicide in the general population $[19,20]$. Precisely, depressive disorders are very highly associated to it, especially in the elderly [21]. Similarly, bipolar disorder involves a high risk for accomplished suicide, and is commonly associated with a previous history of suicide attempts [22,23]. Also, patients suffering from schizophrenia and other psychotic spectrum disorders are at an increased risk of suicide compared to the general population, especially in the early stages of the illness [24-26].

Nevertheless, despite improved knowledge of the risk and protection factors for suicidal behavior, and even though intervention in suicide risk is considered a public health priority, still today, $90 \%$ of the individuals that die by suicide seek help from the healthcare system, mainly resorting to primary care, within the three months before their death [27]. In general, healthcare professionals seem to be fully informed about suicidal behavior, although there are certain deficiencies and difficulties that make it hard to achieve higher affectivity [28]. One of the factors that have been considered is the frequently negative attitude of health professionals towards patients with self-destructive behaviors [29]. This is why it is important to learn about health professionals' attitudes towards suicide and its prevention, as well as to consider the interventions or attitudes that these professionals believe most effective.

The aims of this study are to analyze the differences in the perception of suicide prevention of four different groups of health professionals in a region of north-western Spain in order to establish the priorities for reducing the suicide behavior. This is achieved through a qualitative and a quantitative analysis to quantify the qualitative data. The qualitative methodology will be useful for knowing without prejudices the unmet needs for improving the efficacy of suicide prevention and achieving new proposals for the future [30].

This research is framed within the European Regions Enforcing Actions against Suicide, European Project, which involves 11 regions with different experiences working towards achieving progress in the area of suicide prevention in Europe [31].

\section{Methodology \\ - Design}

A research with two different analyses statistical was carried in this study using a mixed method. Initially, a qualitative research by focus groups was carried on for achieving new proposals and ideas about the suicide prevention and detecting unmet needs for it. And subsequently, a quantitative analysis was applied in order to assign a weight the qualitative data previously achieved.

\section{- Inclusion criteria}

A total of 56 participants were recruited according to the following inclusion criteria:

1. Health workers belonging to the four professional groups selected for this study: Psychiatrists, Psychologists, Emergency physicians and Primary Care physicians.

2. Professional experience in the field of suicide.

\section{Age between 18-65 years old.}

\section{- Recruitment}

The design of the sample was structural, according with different healthcare professionals that are important for the targets of the study. The sample population is made up of health professionals engaged in the prevention, care and intervention in suicide behaviors in the healthcare area of the province of Zamora, where the study is based. Sampling was directed, since rather than generalizing in terms of probability, the goal was to capture the diversity of opinions on suicide behavior of the different healthcare professionals to obtain as much discursive space as possible.

The selection of participants was conducted in the healthcare area of Zamora and in the different centers of the Fundación INTRAS (Foundation for research and development in the mental health area). Groups were structured into strata, balanced according to 
the sociodemographic characteristics of the participants from each professional category.

The selection criterion chosen for this intentional sampling was the professional category of the health staff involved in the prevention of suicidal behavior: psychiatry, primary care (primary care network), psychologists/psychiatrists (mental health network) and emergency physicians (emergency care network). In general terms, the primary care network plays a significant role in detecting the risk for suicide, the emergency care network usually intervenes when suicidal behavior takes place and, finally, the mental health network is involved in reducing or eliminating the risk for suicide.

\section{Procedure}

Eight focus groups were used (two for each professional category), with the participation of a total of 12 primary care physicians, 14 emergency care physicians, 17 psychologists and 13 psychiatrists. $70.6 \%$ of the participants were women and $29 \%$ men. The average age of the participants was 41 , and the average number of years of work experience was 14 .

None of chosen persons were meeting the moderator of the focus groups and neither had they known the research objectives of the study. In this sense, it was possible to avoid that the participants were taking a prepared speech. The script of the focus group (FIGURE 1) was created previously by the research team in order to ensure that the obtained information was useful for the investigation. The introduction of the moderator did not determine the course of the discussion and all realized questions were opened.

All focal groups were video and audio recorded, prior informed consent, to gather the information on the views in interaction of the different health professional categories. Each session lasted between 1 and 1.5 hours and meetings ran smoothly and in a very participatory atmosphere that encouraged the subjects to speak freely, expressing their ideas individually and interactively. These speeches were used to describe and interpret the interand intra-professional variations that allow the differentiation of the meaning of prevention of suicidal behavior for each category.

\section{- Analysis}

A mixed method (pluralism and compatibility) combining the formal rigor of quantitative methods and the flexibility of qualitative methods was applied. The "conversion or transformation" mixed model design, which quantifies qualitative data for further analysis was used. The opinions of health professionals on the dimensions that determine the prevention of suicidal behavior were gathered in a first interpretative analysis and, subsequently, a statistical analysis of the most representative demands described in the speech of each category was conducted.

The use of a qualitative methodology enables access to reality without the need for its

\section{Focus groups scrip}

\footnotetext{
- Clinical importance: What do you think about the importance accorded to suicide attempts in the clinical practice? Do you believe it is given a greater importance to other pathologies?

- Prevention: What do you do to prevent suicide behaviors?

- Available resources for the intervention: Which are the existing resources and facilitators for the intervention?

- Difficulties in identifying suicide behaviors: Which are te difficulties to identify suicide behaviors and suicide risk behaviors?
}

- Identification needs: What might be needed to identify suicide behaviors and suicide risk behaviors?

- Difficulties in suicide behaviors intervention: What difficulties exist in the care of this type of behaviors?

- Intervention facilitators: What facilities exist in the care of suicide behaviors?

- Identification of deficiencies in the intervention: What could be done and not done? What should be done to improve what is being done now?

FIGURE 1. Thematic program of focus groups. 
previous categorization. All speech production, which occurred freely and spontaneously, was considered relevant. The analysis of qualitative data consisted of two stages related to different approaches: a first stage to identify discourse positions and a second stage for data reduction, data layout and transformation, achievement of results and verification of the findings.

The sessions, whose thematic layout is shown in the table below, were transcribed using NVIVO 10 software. Once the group interviews had been transcribed, the categorization or transformation of text into data was conducted and encoding or space-text allocation to the corresponding category of the gathered information was carried out. This was also the stage at which the quality criteria of credibility, dependability and conformability of the textual data gathered were ensured.

\section{Results}

\section{Qualitative analysis}

The description of the discursive positions on suicide behavior and the symbolic configurations has been arranged into a conceptual map including 4 main categories or axes and 12 subcategories (FIGURE 2).

When comparing health professional groups according to the encoding obtained for the main categories "Significance of risk behaviors at work level", "Intervention", "Prevention" and "Number of resources") and in line with the chart above, there is evidence that the four healthcare areas participating in this study give greater importance to Intervention in risk behavior, with a total of 852 references (FIGURE 3).

Working towards "Prevention" takes second place as the groups' main concern, with 348 references. However, it should be noted that the area of psychiatry gives greater importance to resources and their availability and accessibility than to the prevention of suicidal behavior, as opposed to the other groups, although it is true that the opinions expressed by psychiatrists on resources and prevention differ in 9 references.

Regarding available resources, a total of 244 references were obtained. It should also be noted that, although significance at work level of suicide attempts obtained the lowest number of references with a total of 41 , its distribution among the different professional areas is homogeneous.

An analysis of the "Intervention" category shows that there are a series of subcategories that take on greater relevance in the different professional groups. The "Difficulties in intervention" subcategory yields the highest number of codifications in the four healthcare professionals groups, therefore proving the most important for healthcare professionals as a whole.

Low levels of training and lack of time for satisfactory intervention is observed among the primary care professionals:

"I think that we not prepared, generally speaking though, and that many cases escape us and end in suicide because we don't realize their importance. We are aware that there are serious rare pathologies, but we lack the training and, probably, the time." (Reference 7 "Difficulties in intervention" - Group 1 Primary Care)

These low levels of training and lack of time for satisfactory intervention would be the reason by which the primary care professionals have more fear of not knowing how to proceed:

"There is always a fear of what one isn't trained for. When you are trained in something,

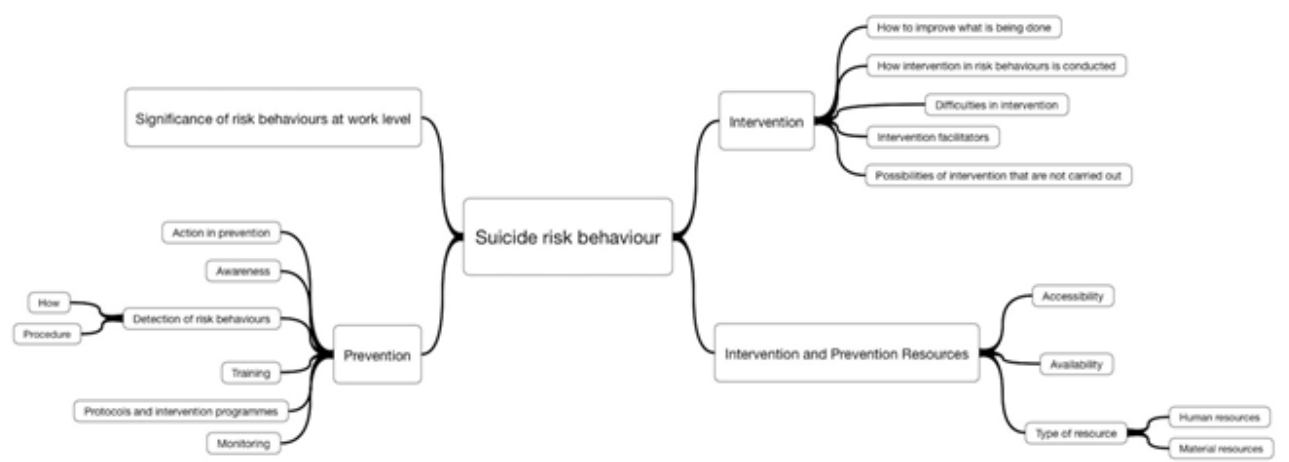

FIGURE 2. Main categories and subcategories of suicide risk behavior significance. 


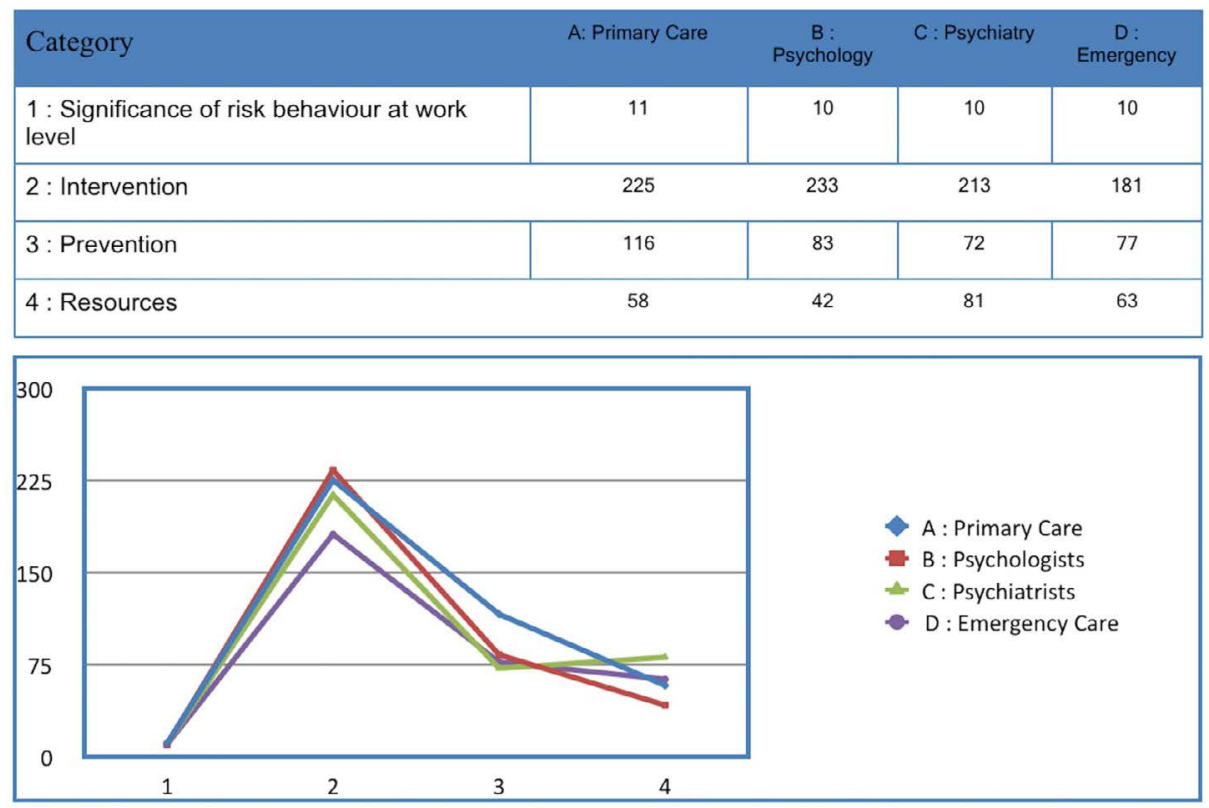

FIGURE 3. Encoded references.

it doesn't frighten you." (Reference 11 "Difficulties in intervention" - Group 1 Primary Care)

Very similar are the considerations observed among professionals working in emergency care, who believe that the greatest difficulty is associated to low levels of training and time constraints:

"I believe that I don't have proper training concerning the Psychiatry to assess many psychiatric patients" (Reference 4 "Difficulties in intervention" - Group 1 Emergency Care)

However, mental health professionals believe that the greatest difficulties are in the management of suicide threats without intent and suicides:

"I believe that ninety percent of what we see is parasuicidal behaviors. It's just that, sometimes, suicidal gestures to achieve a goal can pass to consummation. Therefore, it is very difficult to make the right decision in an emergency, which is when they usually come." (Reference 2 "Difficulties in intervention" Group 1 Psychiatrists).

"I don't call it attention seeking, I call it goaldirected behavior. That is, it is well known that there are patients who know that to mention suicide ideation means that the physician in charge will deploy a full protection system for them. So they can play with that." (Reference
6 "Difficulties in intervention" - Group 1 Psychiatrists).

There also appears to be a certain lack of integration with other participants in the treatment of suicidal behavior:

"I understand that I know suicide from my own patch, which is psychiatry, but, against popular belief, it is a much wider notion." (Reference 14 "Difficulties in intervention" Group 1 Psychiatrists).

Another aspect that is believed to hinder treatment is the lack of continuity of care, hinting that, despite identification, many patients are lost even before treatment is started:

"The problem is in patients' failure to keep appointments." (Reference 51 "Difficulties in intervention" - Group 1 Psychologists)

A suggestion is the creation of more specific and better structured programs to improve treatment:

"What has to be clear is that we don't have any structured and specific prevention programs." (Reference 9 "Difficulties in intervention" Group 2 Psychologists).

On the other hand, the "Intervention facilitators" subcategory ranks second in all professional groups, except for that of emergency physicians. Furthermore, mental health professionals believe in the need for a common 
strategy to be followed by all those involved, and for the possibility to offer alternative ways:

"This is why the patient, the family and we, as the three partners in contact, must be very well informed to be able to notice and detect it". (Reference 32 "Intervention facilitators" Group 1 Psychologists).

"I believe that one of the current problems is that one of the important things at that time is for the individual to see alternative ways, isn't it? An alternative" (Reference 21 "Intervention facilitators" - Group 2 Psychologists).

Mental health professionals also value continuity of care and treatment, either through traditional methods such as community nursing, or through the use of new technologies, as a strategic goal to be achieved:

"I have the peace of mind of knowing that a nursing staff exits. I know that if one day I cannot see the patient, a nurse can go to his house to check how the patient is feeling." Reference 24 "Intervention facilitators" - Group 1 Psychiatrists)

Similarly, primary care professionals believe that a good patient-therapist relationship is crucial for early detection and timely intervention:

"There is trust between me and my patients to create spaces for communication and speak about where we stand at the time, what's going on in their heads and how we see it. And if I detect highly structured self-destructive ideas, I know how to deal with it." (Reference 3 "Intervention facilitators" - Group 1 Primary Care)

"Patient-physician relationship is important, but not only when treating physical problems, it is even more important when it comes to treating mental problems." (Reference 5 "Intervention facilitators” - Group 1 Primary Care)

"How intervention in risk behaviors is conducted" is the second subcategory in number of coding's for the emergency physicians group.
The contents of this subcategory are related to courses of action when the behavior has already taken place:

"Our task is more concerned with the organic, the pressures they have. The psychiatric aspect comes if another attempt takes place or is going to take place, if the patient is at risk or not or whatever, we always pass the case to the psychiatrists." (Reference 1 "How intervention in risk behaviors is conducted" - Group 1 Emergency physicians)

"Here we mainly keep the patient under observation for a while and then ultimate responsibility is always the assumed by the psychiatrist, in terms of whether or not the person might repeat the act. It is not our responsibility, it is the psychiatrist's." (Reference 2 "How intervention in risk behaviors is conducted" - Group 1 Emergency physicians)

\section{Quantitative analysis}

The qualitative analysis conducted shows the opinions of health professionals on the prevention of suicidal behavior. This was followed by a quantitative study where the most representative demands described in each group's discourse were analyzed using simple correspondence analysis. The aim of this technique is "data reduction to determine relationship structures among non-metric variables" such as the ones involved in this study.

\section{Correspondence analyses among the different health professional groups}

The correspondence analyses (TABLE 1) have been performed among the different health professional groups using different categories of suicide risk behavior significance. The simple correspondence analysis determines the distance between variables based on the contingency table with the observations gathered from the focus groups. Reference counting shows proximity among them in the graphs and the degree of association is reflected by proximity or distance. It should be noted that all the

\begin{tabular}{|c|ccccc|}
\hline $\begin{array}{c}\text { Table 1. Contingency table. } \\
\text { Group }\end{array}$ & \multicolumn{7}{c|}{ Category (absolute frequencies) } \\
\hline & Significance at work level & Intervention & Prevention & Resources & Active margin \\
Primary Care & 11 & 263 & 129 & 65 & 468 \\
Psychologists & 10 & 269 & 90 & 52 & 421 \\
Psychiatrists & 10 & 249 & 85 & 91 & 435 \\
Emergency Care & 10 & 227 & 90 & 394 & 419 \\
Active margin & 41 & 1008 & 300 & 1743 \\
\hline
\end{tabular}




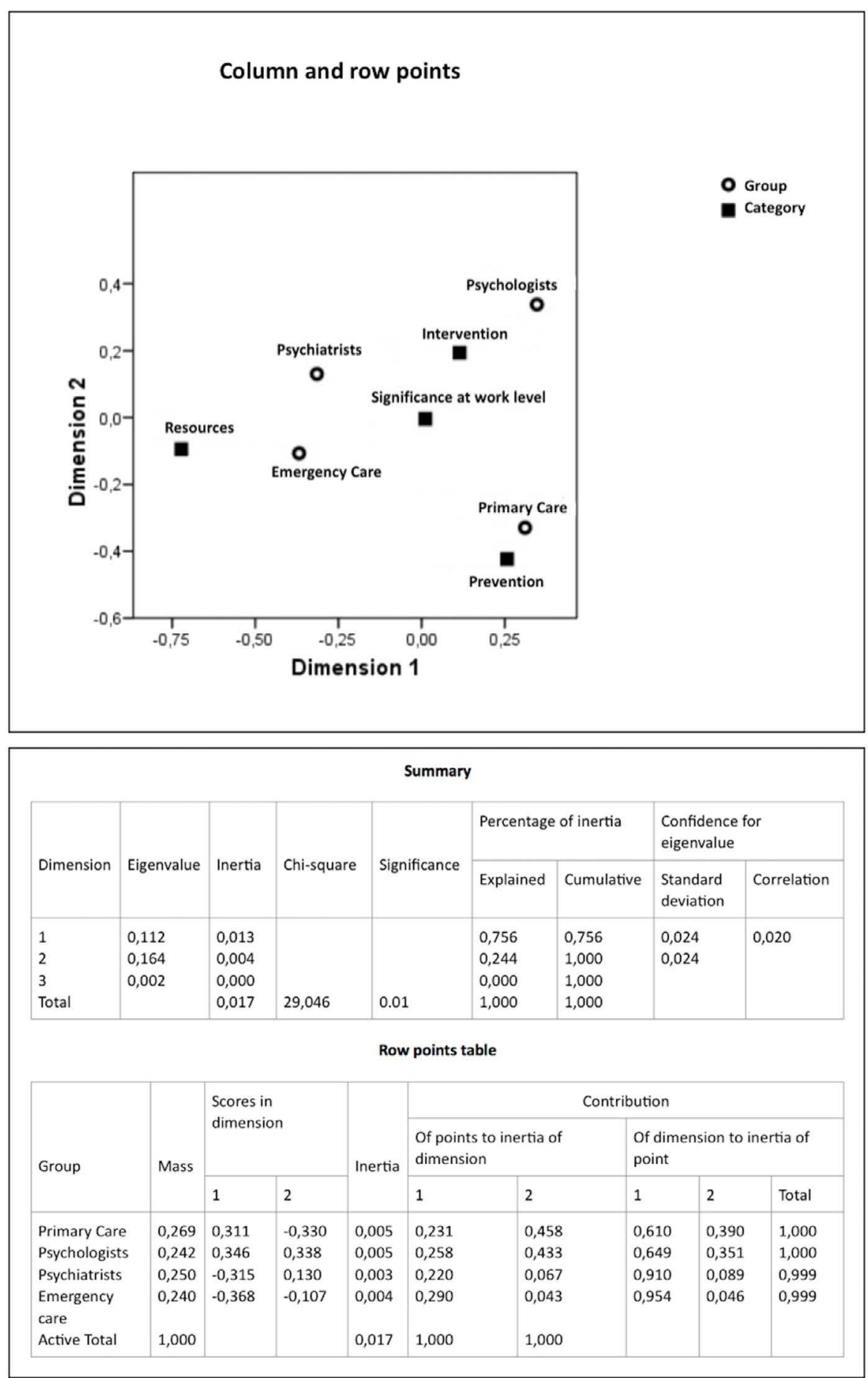

FIGURE 4. Perceptual map.

analyses conducted are significant below the $2 \%$ significance level.

\section{Pattern of association between the} health staff groups and the main categories of suicide risk behavior

From the information of the correspondence analyses it is obtained a perceptual map (FIGURE 4) that place the categories of the variables in two-dimensional space, so that it is possible to determine their degree of association from the distances between them. The perceptual map situates the categories of the variables in two-dimensional space, so that it is possible to determine their degree of association from the distances between them. The pattern of association between the health staff groups and the categories of intervention in 
risk behavior, prevention activities, relevance of these behaviors at work level, and intervention and prevention resources displays four groups that are distant from each other and clustered into two clearly differentiated dimensions. This proximity or distance reflects the association grade, placing in a multidimensional space the categories of the variables.

A configuration of four distant groups is obtained into the perceptual map distributed in both dimensions:

- Group 1: Psychologists - Intervention

- Group 2: Psychiatrists - Significance at work level

- Group 3: Emergency Care - Resources

- Group 4: Primary Care - Prevention

The dimension 1 with greater explanatory weight sets aside the opinions expressed by psychologists (concern about intervention in suicide-risk behaviors) from the other three groups. Results show that physicians working in primary care are more interested in prevention, emergency physicians in available resources and psychiatrists in the relevance of this type of behaviors at the work level. The dimension 2 with less explanatory weight sets primary and emergency care physicians apart from the other two.

Both dimensions are further identified in a similar way to how the factor analysis is conducted. In Dimension 1, a clear separation of the cases can be observed: Primary Care and Psychologists appear in the positive pole, whereas Psychiatrists and Emergency Care appear in the negative pole. In Dimension 2, the cases of Primary Care (negative pole) are clearly positioned at a distance from the rest (positive pole).

The mass contains the proportion of cases of the category out of the total. If it is interpreted as a percentage it can be stated that out of the $100 \%$ cases that make up the sample, Primary Care accumulates $26.9 \%$, Psychiatrists 25\%, Psychologists 24.2\% and Emergence Care 24\%. Inertia reports the weights of the categories in the dimensions and of the dimensions in the categories. The test table corresponding to the row points shows the weight of each dimension in the categories. The categories of Psychiatrists and Emergency Care (0.910 and 0.954) in Dimension 1 show the highest weights, both with a negative score. On the other hand,
Primary Care and Psychologists (0.390 y 0.351) are those with the highest weights in Dimension 2 , scoring positively against the other two categories.

To sum up, the opinions of Psychiatrists and Emergency Care physicians are close to each other in the two-dimensional space, as are those of Primary Care physicians and Psychologists, although the distance between the opinions of the two latter groups is shorter than that between the two others. If all four groups are compared at the same time, the position of Primary Care physicians is the most distant, focusing mainly on prevention issues.

\section{Discussion}

The correspondence analysis conducted yields variable groups that are interesting from the healthcare perspective for improving the organization of the care of people at risk for suicide and the prevention of suicidal behaviors.

Psychiatrists seem to believe that addressing suicidal behavior is one of the most relevant aspects of their everyday clinical practice, since it ranks first in psychiatric emergencies at general hospitals and because failure to identify the risk for suicide, or not taking the required intervention measures, may result in the patient's death. According to Rothes, although patient suicide is a relatively frequent phenomenon in a psychiatrist's clinical practice, this does not make it less traumatic for these professionals [32]. A study conducted by Thomyangkoon on the impact of patients death by suicide on a sample of psychiatrists concluded that over $50 \%$ developed feelings of sadness, guilt or hopelessness [33]. The psychiatrists in this study believe that the greatest difficulty they face in the treatment of patients with selfharming behaviors is in differentiating selfdestructive attempts with suicidal purposes from those with a manipulative purpose used by patients as a tool to achieve a goal. Thus, for psychiatrists, intervention difficulties mainly lie in formulating a differential diagnosis of suicidal behavior. An additional problem among these professionals is the fact that a number of the patients that show suicidal behaviors never come into contact with a psychiatrist, either because they are people from a disadvantaged socioeconomic environment and do not have a history of mental illness, or because the act has already been committed before being referred to the mental health network. 
In this regard, emphasis should be placed on the point of view of the different mental health professionals, while psychiatrists' main concern is early detection and diagnosis of the risk for suicide and to address it in its correct clinical dimension, psychologists believe the main difficulties lie in direct intervention once such risk has been identified. Thus, psychologists observe a lack of intervention programs or plans in the area of suicidal behavior. Similarly, the psychologists-intervention correlation confirms the strong need for launching specific psychological treatment for suicide prevention programs. These results reflect the deficiencies in specific psychological intervention in the area of suicidal behavior and the fact that professionals in the field of psychology themselves recognize this problem. In this sense, it should be noted that there are psychological treatment interventions that have proved positively effective in the prevention of suicidal behavior [34-36], although there are authors such as Fowler who do not agree [37]. This professional group also points out work on the patient's environment as a facilitator for intervention in suicidal behavior, which involves a psychosocial approach to treatment that is especially relevant for the development of a bio-psychosocial treatment model. On the other hand, the fact of being able to offer life projects to patients with suicidal behavior as a part of therapeutic intervention is also particularly interesting.

Likewise, psychologists' role should be considered highly important both in high risk cases and in those where there is a greater manipulative component, since their scope of action goes beyond the mental illness, unlike psychiatrists, whose action is mainly targeted at the mental illness itself. Another relevant aspect is the use of new technologies as facilitators for intervention, opening the possibility of increased access for certain individuals who are reluctant to seeking help from mental health services. Finally, attention should be drawn to the importance given to the existence of community nursing professionals involved in addressing suicidal behavior, since they make intervention on these patients easier and enable closer monitoring of these cases. This aspect is more positively valued by the psychiatrists, who mention lack of sustained care and monitoring as one of the main problems in addressing suicidal behaviors.

The emergency care physicians stated that the presence of a psychiatrist that can assess the risk for suicide is crucial to the Emergency Care Unit, since they believe it ensures safety [38]. However, the performance of emergency care physicians and their assessment of the patient are extremely valuable, since they are the first to provide aid for patients that have attempted or are at risk for suicide [39]. Nevertheless, according to the results obtained from this study, these professionals do not perceive their role in prevention and care of suicidal behavior as relevant, claiming that their performance is reduced to treating the physical pathology causing the risk for suicide, which does not include psychiatric assessment. In this regard, these professionals state that they lack sufficient skills and knowledge to make decisions in the treatment of suicidal behaviors, so that they leave all responsibility for the treatment of these emergency care patients to psychiatrists. Therefore, it is necessary to improve the training of this professional group in early detection and management of suicidal behaviors. In the same vein, the Emergency Care-Resources correlation proves the importance that these professionals attach to the availability of appropriate resources for the correct treatment of patients at risk for suicide, with special emphasis on easy access to seek emergency psychiatric assessment at any time. These results, which show emergency care physicians' lack of involvement in the treatment and prevention of suicidal behaviors, explain why this is the only group of the four where identification of facilitators in intervention lacks significant weight.

Tait believes that, because primary care physicians are those in closest contact with patients during the first stages of development of suicidal ideation, they play a major role in early detection and prevention of suicidal behaviors [40]. The physicians belonging to this group that have taken part in this study point out the lack of time and sufficient knowledge to address such behaviors as the two key aspects that hinder their performance in this area. Nevertheless, they attach a great deal of importance to a good patient-physician relationship and the need for close contact with patients, two aspects they believe to possess and which contribute to early detection of the risk for suicide. A study conducted by Saini reflects difficulties for this group of professionals in addressing suicidal behaviors, the results showing that most primary care doctors consider that they lack the training required to properly identify risk for suicide, and that find difficulties when it comes 
to referring patients to Mental Health services [41]. According to Redsch, it is common among primary care physicians to request more extensive training in mental health care, especially in the identification, management and treatment of patients at risk for suicide [42]. The Primary Care- prevention correlation yielded by the qualitative analysis conducted in this study also reflects this picture of difficulties in addressing suicidal behaviors and the need for further training in this area. Thus, these results raise awareness of the current need for creating specific training programs for Primary Care in the area of suicide.

On the other hand, the two-dimensional space analysis shows a clear distinction between the opinions of psychiatrists and emergency care physicians, and those of psychologists and primary care physicians, as clearly illustrated by graph of the (FIGURE 3). The statistical analysis of the opinions of psychiatrists and emergency care physicians are also more significant in dimension 1 . These differences in the opinions of professional groups can be explained by their different forms of clinical contact with suicidal patients. Psychiatrists and emergency care physicians usually see these patients in times of crisis, while primary care physicians and psychologists do so in the earliest stages of development of self-destructive ideas or when they have survived a suicide attempt. In this regard, and as shown by graph of the (FIGURE 3), the small importance attached to resources by primary care physicians and psychologists it is worth pointing out. This result is along the same lines in the differences in the form of contact with suicidal patients among these four groups, given that the greatest need for resources is in situations that require immediate intervention in suicidal behavior. Thus, primary care physicians and psychologists attach higher relevance to everything related to prevention of suicidal behaviors and how to address them, while psychiatrists and emergency care physicians believe that all aspects are considerably relevant, giving major importance to correct identification, and a little less to prevention. This could be due to the fact that the latter generally work with patients whose condition is more severe, meaning that suicidal behavior is already fully developed, so that their intervention is more focused on crisis management and resolution.

The limitations of the study are related to the geographical area in which have been made this work. All of workers are involved in the health network of Zamora and so, many of the results and points of view of the participants can be influenced by the specific features of the mental health network of Zamora. However, there are few differences between the statistics of suicide in Zamora vs. the others health areas of Spain, and there aren't specific features of the mental health network. Likewise, the workers involved in the study have not chosen or recruited in a randomized way, but taking into account the type of analysis (qualitative) was more important to choose people representative of all points of view of the health system and obtain proposals from them, than do the study with a representative sample. The interest was to know the different points of view of the health workers about the unmet needs and proposals for improving and so, the recruitment was addressed for achieving this goal.

\section{Acknowledgments}

The authors thank all those who participated in this study and all the members of the Euregenas Project (No. 2010-12-03). The Euregenas Project has received funding from the European Union under the Public Health Program 2008-20135. 


\section{REFERENCES}

Chen IM, Liao SC, Lee MB, et al. Risk factors of suicide mortality among multiple attempters: A national registry study in Taiwan. J. Formos. Med. Assoc. 115(5), 364-371 (2015).

WHO, Suicide Prevention (SUPRE). 2014, World Health Organization: Geneva.

Fond G, Zendjidjian X, Boucekine $\mathrm{M}$ et al. The World Health Organization (WHO) dataset for guiding suicide prevention policies: A 3-decade French national survey. J. Affect Disord. 188, 232-238. (2015).

Milner A, Page K, Spencer-Thomas S, Lamotagne AD. Workplace suicide prevention: a systematic review of published and unpublished activities. Health Promot. Int. 30(1), 29-37 (2015).

Malakouti SK, Nojomi M, Poshtmashadi $\mathrm{M}$, et al. Integrating a suicide prevention program into the primary health care network: a field trial study in Iran. Biomed. Res. Int. 193729 (2015).

Eynan R, Reiss L, Links P, et al. Suicide prevention competencies among urban Indian physicians: A needs assessment. Indian J. Psychiatry. 57(4), 397-402 (2015).

de Beurs DP, de Groot MH, de Keijser J, et al. The effect of an e-learning supported Train-the-Trainer programme on implementation of suicide guidelines in mental health care. J. Affect Disord. 175, 446-53 (2015).

Lai MH, Maniam T, Chan LF, Ravindran AV. Caught in the web: a review of web-based suicide prevention. J. Med. Internet Res. 16(1), e30 (2014).

Shand FL, Ridani R, Tighe J, Christensen $H$. The effectiveness of a suicide prevention app for indigenous Australian youths: study protocol for a randomized controlled trial. Trials. 14, 396 (2013).

Sueki H, Ito J. Suicide prevention through online gatekeeping using search advertising techniques: a feasibility study. Crisis. 36(4), 267-273 (2015).

Whiteside U, Lungu A, Richards J, et al. Figure correction: Designing messaging to engage patients in an online suicide prevention intervention: survey results from patients with current suicidal ideation. J. Med. Internet Res. 17(4), e69 (2015).

Thompson AH, Dewa CS, Phare S. The suicidal process: age of onset and severity of suicidal behaviour. Soc. Psychiatry Psychiatr. Epidemiol. 47(8), 1263-1269 (2012).

Runeson BS, Beskow J, Waern M. The suicidal process in suicides among young people. Acta Psychiatr. Scand. 93(1), 35-42 (1996).

Isometsa ET, Lonnqvist JK. Suicide attempts preceding completed suicide. Br. J. Psychiatry 173, 531-535 (1998).

Neeleman J, de Graaf R, Vollebergh W. The suicidal process; prospective comparison between early and later stages. J. Affect Disord. 82(1), 43-52.

Ashrafioun L, Pigeon WR, Conner KR, et al. Prevalence and correlates of suicidal ideation and suicide attempts among veterans in primary care referred for a mental health evaluation. J. Affect Disord. 189, 344-350 (2016).

Congdon P. Latent variable model for suicide risk in relation to social capital and socio-economic status. Soc. Psychiatry Psychiatr. Epidemiol. 47(8), 1205-1219 (2012).

Roy A. Gene-Environment Interaction and Suicidal Behavior, The Neurobiological Basis of Suicide, Dwivedi Y, Editor. 2012: Boca Raton (FL).

Cavanagh JT, Carson AJ, Sharpe M, et al. Psychological autopsy studies of suicide: a systematic review. Psychol. Med. 33(3), 395-405 (2003).

Osborn D, Levy G, Nazareth I, et al. Suicide and severe mental illnesses. Cohort study within the UK general practice research database. Schizophr. Res. 99(1-3), 134-138 (2008).

Yoshimasu K, Kiyohara C, Miyashita K.
Suicidal risk factors and completed suicide: meta-analyses based on psychological autopsy studies. Environ. Health Prev. Med.. 13(5), 243-256 (2008).

Costa Lda S, Alencar ÁP, Neto NPJ et al. Risk factors for suicide in bipolar disorder: a systematic review. J. Affect Disord. 170, 237-254 (2015).

Hawton K, Sutton L, Haw C, Sinclair $\mathrm{J}$, et al. Suicide and attempted suicide in bipolar disorder: a systematic review of risk factors. J. Clin. Psychiatry. 66(6), 693-704 (2005).

DeVylder JE, Lukens EP, Link BG, et al. Suicidal ideation and suicide attempts among adults with psychotic experiences: data from the Collaborative Psychiatric Epidemiology Surveys. JAMA Psychiatry 72(3), 219-225 (2015).

Palmer BA, Pankratz VS, Bostwick JM. The lifetime risk of suicide in schizophrenia: a reexamination. Arch. Gen. Psychiatry. 62(3), 247-253 (2005).

Tandon R. Suicidal behavior in schizophrenia. Expert Rev. Neurother. 5(1), 95-99 (2005).

De Leo D, Draper BM, Snowdon J, et al. Contacts with health professionals before suicide: missed opportunities for prevention? Compr. Psychiatry. 54(7), 1117-1123 (2013).

Smith AR, Silva C, Covington DW, Joiner TE. An assessment of suicide-related knowledge and skills among health professionals. Health Psychol. 33(2), 110-119 (2014).

Saunders KE, Hawton K, Fortune S, et al. Attitudes and knowledge of clinical staff regarding people who self-harm: a systematic review. J. Affect Disord. 139(3), 205-216 (2012).

Stanistreet DG, Gabbay MB, Jeffrey $\mathrm{V}$, et al. The role of primary care in the prevention of suicide and accidental deaths among young men: an epidemiological study. Br. J. Gen. Pract. 54(501), 254-258 (2004). 
Euregenas. European Regions Enforcing Actions Against Suicide. Available from: http://www.euregenas.eu/

Rothes IA, Scheerder G, Van Audenhove C, et al. Patient suicide: the experience of Flemish psychiatrists. Suicide Life Threat Behav. 43(4), 379394 (2013).

Thomyangkoon P, Leenaars A. Impact of death by suicide of patients on Thai psychiatrists. Suicide Life Threat Behav. 38(6), 728-740 (2008).

Angeletti G, Pompili M, Innamorati M, et al. Short-term psychodynamic psychotherapy in patients with "male depression" syndrome, hopelessness, and suicide risk: a pilot study. Depress. Res. Treat. 2013, 408983 (2013).

Heisel MJ, Talbot NL, King DA, Tu XM. Adapting interpersonal psychotherapy for older adults at risk for suicide. Am. J. Geriatr. Psychiatry. 23(1), 87-98 (2015).
Hepp U, Wittmann L, Schnyder U, Michel K. Psychological and psychosocial interventions after attempted suicide: an overview of treatment studies. Crisis. 25(3), 108-117 (2004).

Fowler JC. Review: No reliable evidence of the effect of psychotherapy upon suicide risk in people with depression. Evid. Based Ment. Health. 16(3), 73 (2013).

Suokas JK, Suominen, Lonnqvist J. The attitudes of emergency staff toward attempted suicide patients: a comparative study before and after establishment of a psychiatric consultation service. Crisis. 30(3), 161-165 (2009).

Cooper JB, Lawlor MP, Hiroeh U, Kapur N, Appleby L. Factors that influence emergency department doctors' assessment of suicide risk in deliberate self-harm patients. Eur. J. Emerg. Med. 10(4), 283-287 (2003).
Tait L, Michail M. Educational interventions for general practitioners to identify and manage depression as a suicide risk factor in young people: a systematic review and meta-analysis protocol. Syst. Rev. 3, 145 (2014).

Saini P, Windfuhr K, Pearson A, et al. Suicide prevention in primary care: General practitioners' views on service availability. BMC Res. Notes. 3, 246 (2010).

Redsch O, Miyaishi S, Heinemann A, et al. Comparison of German and Japanese general practitioners' awareness of suicide and attitudes toward patients with suicidal ideation. Acta Med. Okayama. 60(3), 159-165 (2006). 